\title{
Frustrated Interpersonal Needs and Life Satisfaction in the Elderly Living Alone
}

\author{
Seong Hwan Kim, MD, PhD, ${ }^{1}$ Ji Yoon Park, $\mathrm{MD},{ }^{1}$ and Jae Hong Park, $\mathrm{MD}, \mathrm{PhD}^{1,2}$ \\ Department of Psychiatry, School of Medicine, Dong-A University, Busan, Korea ${ }^{1}$ \\ Busan Regional Mental Health Welfare Center, Busan, Korea ${ }^{2}$
}

\section{Objective}

This study aims to investigate differences between the elderly living alone and those living with others in frustrated interpersonal needs and life satisfaction. Also, we explore the effects of frustrated interpersonal needs on life satisfaction in the elderly living alone.

\section{Method}

The participants include 572 elderly people aged over 60 in Busan, Korea. Life satisfaction were evaluated by the Life Satisfaction Self-Rating Scale (LSRS) and frustrated interpersonal needs were measured by the Interpersonal Needs Questionnaire-Revised (INQ-R).

\section{Result}

The overall LSRS scores were significantly lower in the elderly living alone $(106.2 \pm 20.1)$ than living with others (115.3 $\pm 13.9, \mathrm{p}<0.001)$. The total INQ $-\mathrm{R}$ score was significantly lower in the elderly living alone $(73.8 \pm 12.1)$ than those living with others $(76.9 \pm 12.0, \mathrm{p}<0.05)$. As a result of multiple regression analysis, we came to know that frustrated interpersonal needs were factors affecting life satisfaction in living alone group.

Table 1. General characteristics of participants

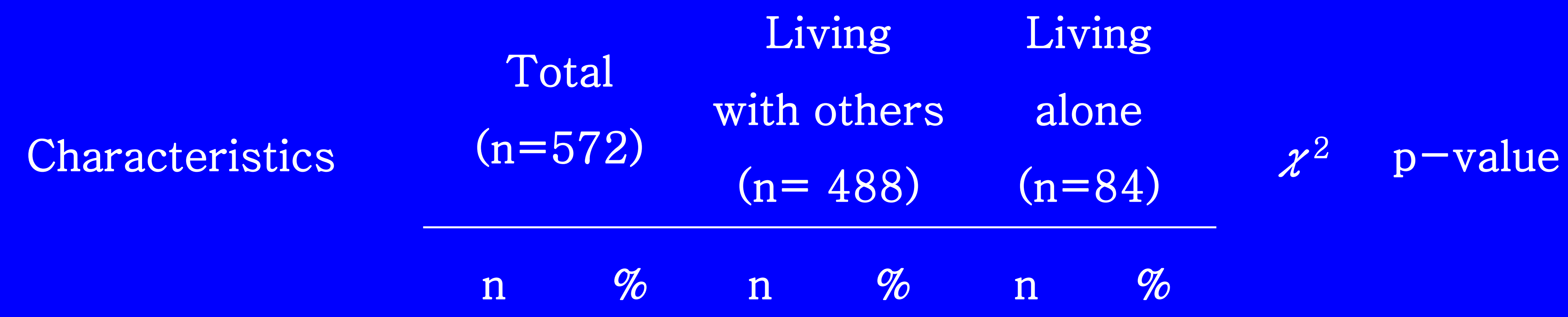

Sex

Male

$\begin{array}{llllll}256 & 44.8 & 226 & 46.3 & 30 & 35.7\end{array}$

Female

$\begin{array}{llllll}316 & 55.2 & 262 & 53.7 & 54 & 64.3\end{array}$

Education

Less than middle

\begin{tabular}{ll|llll}
289 & 50.5 & 224 & 45.9 & 65 & 77.4
\end{tabular}

school graduate

$\begin{array}{llllllll}\text { High school graduate } & 247 & 43.2 & 229 & 46.9 & 18 & 21.4\end{array}$

$\begin{array}{llllllll}\text { More than college } & 36 & 6.3 & 35 & 7.2 & 1 & 1.2\end{array}$

graduate

Religion

1.5110 .219

Yes

No

Occupation

Yes

No

Income $(1,000$ won $)$

$<2,000$

$2,000-4000$

$4,000-6,000$

$\geq 6,000$

Age $($ mean $\pm S D)$

years*
Table 2. Difference between life satisfaction and frustrated interpersonal needs in the elderly living with others or those living alone

\begin{tabular}{|c|c|c|c|c|c|c|}
\hline & \multicolumn{2}{|c|}{$\begin{array}{l}\text { Living with } \\
\text { others } \\
(n=488)\end{array}$} & \multicolumn{2}{|c|}{$\begin{array}{l}\text { Living } \\
\text { alone } \\
(\mathrm{n}=84)\end{array}$} & \multirow[t]{2}{*}{$\mathrm{t}$} & \multirow[t]{2}{*}{$\mathrm{p}$-value } \\
\hline & Mean & $\mathrm{SD}$ & Mean & $\mathrm{SD}$ & & \\
\hline \multicolumn{7}{|l|}{ Life satisfaction } \\
\hline Food, clothing, and shelter & 18.1 & 2.3 & 16.6 & 3.6 & 3.543 & 0.001 \\
\hline Friendship & 14.1 & 2.3 & 13.0 & 3.1 & 3.071 & 0.003 \\
\hline Family and relatives & 10.5 & 1.7 & 9.9 & 2.1 & 2.848 & 0.005 \\
\hline Physical and mental health & 17.4 & 2.4 & 16.5 & 2.9 & 2.821 & 0.005 \\
\hline Job & 7.0 & 1.2 & 6.2 & 1.4 & 4.920 & $<0.001$ \\
\hline Economy & 6.6 & 1.4 & 5.6 & 1.8 & 4.532 & $<0.001$ \\
\hline Human relationship & 6.9 & 1.3 & 6.3 & 1.4 & 3.711 & $<0.001$ \\
\hline $\begin{array}{l}\text { Social activities and leisure } \\
\text { activities }\end{array}$ & 13.8 & 2.2 & 12.9 & 2.8 & 2.831 & 0.006 \\
\hline Happiness & 10.3 & 1.7 & 9.3 & 2.1 & 4.559 & $<0.001$ \\
\hline Autonomy and self-esteem & 10.3 & 1.6 & 9.5 & 1.9 & 4.399 & $<0.001$ \\
\hline Total & 115.3 & 13.9 & 106.2 & 20.1 & 3.992 & $<0.001$ \\
\hline \multicolumn{7}{|l|}{ Frustrated interpersonal needs } \\
\hline Perceived burdensomeness & 34.0 & 6.2 & 32.5 & 6.0 & 2.033 & 0.043 \\
\hline Low belongingness & 42.8 & 7.2 & 41.1 & 7.3 & 1.973 & 0.049 \\
\hline Total & 76.9 & 12.0 & 73.8 & 12.1 & 2.235 & 0.026 \\
\hline
\end{tabular}

Table 3. Factors affecting life satisfaction of the elderly living alone

\begin{tabular}{lcccc}
\multicolumn{1}{c}{ Variables } & B & S.E & $\beta$ & p-value \\
Perceived burdensomeness & 0.796 & 0.096 & 0.324 & $<0.001$ \\
Low belongingness & 1.150 & 0.072 & 0.548 & $<0.001$
\end{tabular}

Adjusted for Age, Sex, Religion, Occupation, Educational level, Monthly income

\section{Conclusion}

The results of present study suggest that the elderly living alone are more likely to have poor life satisfaction and frustrated interpersonal needs. Also, frustrated interpersonal needs are related to life satisfaction. Thus, it is necessary to establish the social support system such as social isolation prevention and interpersonal relationship development.

†Corresponding author: Jae Hong Park, Department of Psychiatry, School of Medicine, Dong-A University, Busan, Korea.

E-mail :drpark@dau.ac.kr 